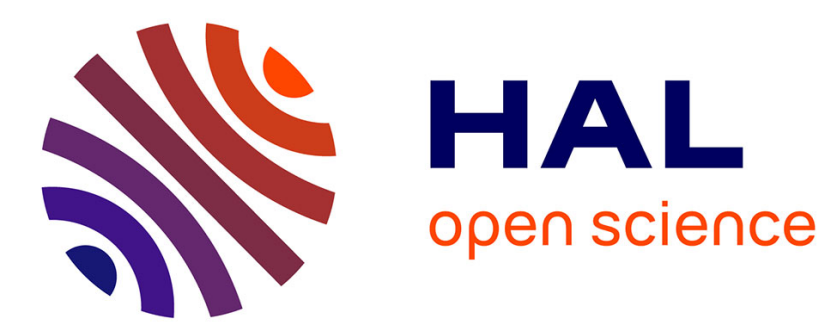

\title{
QUELQUES REFLEXIONS D'ENSEMBLE
}

P.-G. de Gennes

\section{To cite this version:}

P.-G. de Gennes. QUELQUES REFLEXIONS D'ENSEMBLE. Journal de Physique Colloques, 1980, 41 (C3), pp.C3-37-C3-37. 10.1051/jphyscol:1980306 . jpa-00219825

\section{HAL Id: jpa-00219825 https://hal.science/jpa-00219825}

Submitted on 1 Jan 1980

HAL is a multi-disciplinary open access archive for the deposit and dissemination of scientific research documents, whether they are published or not. The documents may come from teaching and research institutions in France or abroad, or from public or private research centers.
L'archive ouverte pluridisciplinaire $\mathbf{H A L}$, est destinée au dépôt et à la diffusion de documents scientifiques de niveau recherche, publiés ou non, émanant des établissements d'enseignement et de recherche français ou étrangers, des laboratoires publics ou privés. 


\author{
QUELQUES REFLEXIONS D'ENSEMBLE \\ P.G. de GENNES \\ Cozlège de France, 75231 Paris Cedex 05, France
}

Nous avons vu un film très suggestif et très soigné sur les oscillations temporelles ou spatiales montrées par certains systèmes hors d'équilibre. La présentation suggère que de nombreux phénomènes biologiques seront justiciables d'une description analogue. Mais beaucoup de biophysiciens, dont Guéron est un exemple, critiquent cette attitude. Je crois que ce débat est instructif, au-delà même du problème spécifique qui est discuté ici :

1) Un système traversé par des flux forts peut engendrer des instabilités et des structures spatiales remarquables (1'exemple le plus beau que je connaisse est celui d'un cristal de neige).

2) L'Ecole de Bruxelles, animée par Progogine, a entrevu très tôt la richesse de ces phénomènes (et leur rôle potentiel pour comprendre l'origine de la vie). Elle n'a pas réussi, toutefois, à les faire rentrer dans un véritable corps de doctrine : pas de méthodes gênérales d'approche, pas de classification fine des bifurcations, etc (+)

3) La vaste dimension de ces concepts et le langage support ont fasciné beaucoup de chercheurs, notamment de non physiciens, et on a vu invoquer des structures dissipatives un peu partout : Guéron nous montre sur quelques exemples précis le danger de ces assimilations 1âches.

4) Les situations oũ un concept (un "outil") scientifique est transformé en objet de culte sont assez fréquentes dans l'histoire des sciences (++). Elles sont particulièrement nuisibles dans les domaines interdisciplinaires - à développement fragile. La biophysique a déjà rencontrê un problème de ce genre il y a quelques années, quand certains ont cru que la chimie quantique allait apporter des éléments considérables à la biologie moléculaire : nous savons maintenant que le rôle de la chimie quantique est positif mais assez modeste. Chaque fois, il y a danger de retour en arrière : les collaborations entre biologistes et physiciens ont souffert, à un certain stade, des déceptions suivant la vague de la chimie quantique.

5) Conclusion pratique : si nous, physiciens, voulons faire de la recherche interdisciplinaire, évitons toute attitude triomphaliste. N'essayons pas de plaquer immédiatement sur une réalité encore mal cernée, des concepts et des modèles physiques qui nous sont chers. Apprenons d'abord patiemment le langage des indigènes : nous découvrirons souvent qu'il est plus riche que nous ne l'imaginons (*). Et ensuite seulement, nous pourrons essayer d'y incorporer quelques mots nouveaux.

(+) Ainsi, la notion - essentielle - de régime chaotique est apparue plus tard et dans un contexte très différent, avec les travaux de Ruelle et Takens, May et otter, P. Martin, etc.

$\left.{ }^{++}\right)$Voir P.G. de Gennes : "Sur les erreurs des sciences dites exactes" NRF, à parâtre

(*) Ainsi la détermination de la séquence des bases sur un acide nucléique est maintenant réalisée de façon infiniment plus élégante et rapide par les méthodes biochimiques que par tout l'arsenal physique! 